\title{
Uncommon Bone Metastases of the Hand
}

\author{
Sarah Houimli' ${ }^{1}$, Mohamed Amine Selmene ${ }^{2}$ \\ ${ }^{1}$ Plastic, Reconstructive and Aesthetic Surgery Department, Trauma Center, Ben Arous, Tunisia \\ ${ }^{2}$ Orthopedic Surgery Department, Trauma Center, Ben Arous, Tunisia \\ Email: sarah.houimli@gmail.com, selmene.amine@gmail.com
}

How to cite this paper: Houimli, S. and Selmene, M.A. (2020) Uncommon Bone Metastases of the Hand. Open Journal of Orthopedics, 10, 395-402.

https://doi.org/10.4236/ojo.2020.1012037

Received: October 8, 2020

Accepted: December 20, 2020

Published: December 23, 2020

Copyright $\odot 2020$ by author(s) and Scientific Research Publishing Inc. This work is licensed under the Creative Commons Attribution International License (CC BY 4.0).

http://creativecommons.org/licenses/by/4.0/ (c) (i) Open Access

\begin{abstract}
Bone metastases are common and occur in several types of cancer. But when they occur in rare localizations, such as in the hand, they can be the source of a diagnostic and prognostic problem. We report two cases of bone metastases occurring in the hand. The objective of our work is to make the reader think of the diagnosis of metastases in front of a bone lesion in the hand despite its rarity and its occurrence in a patient without pathological history or is followed-up for a cancer which was declared cured or under treatment. Both patients presented with painful swelling, the first one at the $3^{\text {rd }}$ finger and the other one at the $3^{\text {rd }}$ metacarpal. In the first case, bone metastasis revealed the existence of the cancer of origin, and in the second, it was diagnosed in a known patient with cancer. Bone biopsy and histological examination confirmed the diagnosis. Treatment at this advanced stage of the cancerous disease is almost always palliative because, in part, of the aggressiveness of bone metastases. A rapid diagnosis of acrometastases is mandatory in order to avoid a delay in treatment. They are characterized by a pejorative prognosis. Pain control is essential in these types of patients.
\end{abstract}

\section{Keywords}

Hand, Bones, Metastases, Surgery

\section{Introduction}

Bone metastases are not uncommon. They occupy the $4^{\text {th }}$ position after the lymph node, pulmonary and hepatic localization in terms of frequency. They are recognized by their poor prognosis and difficult management. They more frequently affect the spine, the pelvis and long bones (humerus, femur). However, they can have rare localizations causing a difficulty and a delay in the diagnosis and the treatment.

We report two cases of bone metastases in the hand which is both a rare loca- 
lization and characterized by a late onset in cancer disease.

\section{Case Report}

These are two cases of bone metastases to the hand, the history of which differed from patient to patient.

The first case was a 52-year-old man, smoking 20 packs/year, who consulted for a painful swelling in the first phalanx (P1) of the 3rd right finger. It was $2 \mathrm{~cm}$ long and had been evolving for 2 months. Radiography showed a well-defined bone lysis at the distal diaphysis-metaphysis junction of $\mathrm{P} 1$ of the $3^{\text {rd }}$ finger with rupture of the cortex in some places (Figure 1). An ultrasound was requested and was in favor of an enchondroma. Immobilization combined with analgesic treatment and additional MRI exploration was initially indicated. The rapid worsening after a week marked the development with the appearance of severe edema of the back of the hand, loss of both appetite and weight. The radiological control showed a total lysis of the cortex (Figure 2). The MRI concluded that there was a single blowing lesion driving back the adjacent structures in favor of acrometastases (Figure 3). The chest X-ray was without abnormalities. A thoraco-abdomino-pelvic computed tomography (TAP CT) scan was requested as part of the extension workup and showed a suspicious parenchymal mass in the right pulmonary apex. The bone biopsy was performed and the pathology examination concluded to a bone metastasis of a moderately differentiated adenocarcinoma (ADK) (Figure 4, Figure 5). The patient had an amputation of his $3^{\text {rd }}$ finger and was referred to the oncology department where palliative treatment for pulmonary ADK was initiated. The patient died after 5 months.

The second case was a 76-year-old man, operated on 7 years ago for, a rectal adenocarcinoma with simple consequences and without any other pathological history. He consulted for a painful swelling of the distal half of the $3^{\text {rd }}$ right metacarpal (M3). The hand X-ray showed bone lysis with complete rupture of the cortex (Figure 6, Figure 7). The MRI concluded to an aspect consistent with acrometastases (Figure 8). As part of the extension assessment, the bone scan showed multiple bone metastases: sternal, costal, sacroiliac, etc. TAP CT scan showed a secondary lingual nodule with osteolytic lesion of the $3^{\text {rd }}$ rib. He had a bone biopsy. The pathology examination concluded to the existence of a bone metastasis of an ADK compatible with the primitive rectum (Figure 9, Figure 10). The patient was at high anesthetic risk and surgery could not be done. $\mathrm{He}$ was sent to oncology for additional treatment (radiotherapy). He died after 4 months.

\section{Discussion}

Neoplastic spread to the bones of the hand is rare. Its frequency is estimated at $0.1 \%$ according to the majority of authors $(0.007 \%$ to $0.3 \%)$ [1].

Keramidas [2] reported that $\mathrm{Wu}$ [3] and Amadio [4] respectively described 3 and 9 cases of localized metastases in the bones of the hand from 41,833 and 


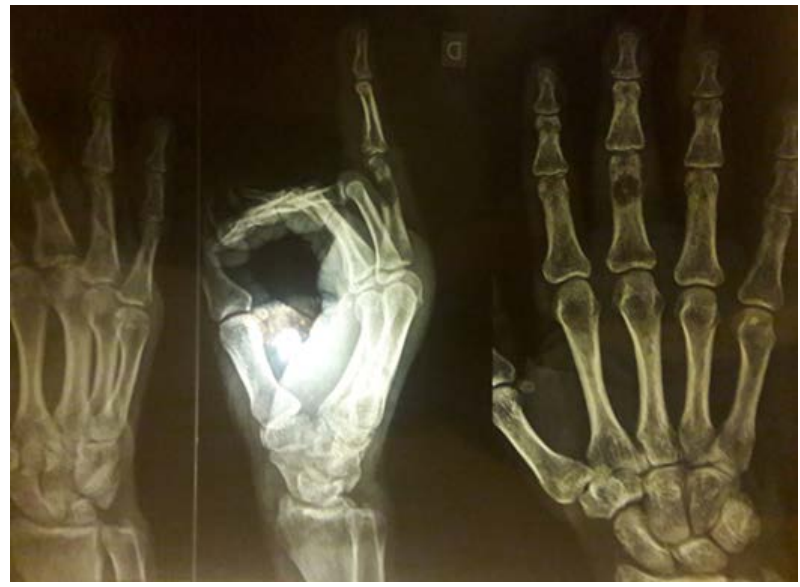

Figure 1 . Hand $\mathrm{x}$-ray of the $1^{\text {st }}$ patient at the $1^{\text {st }}$ consultation.

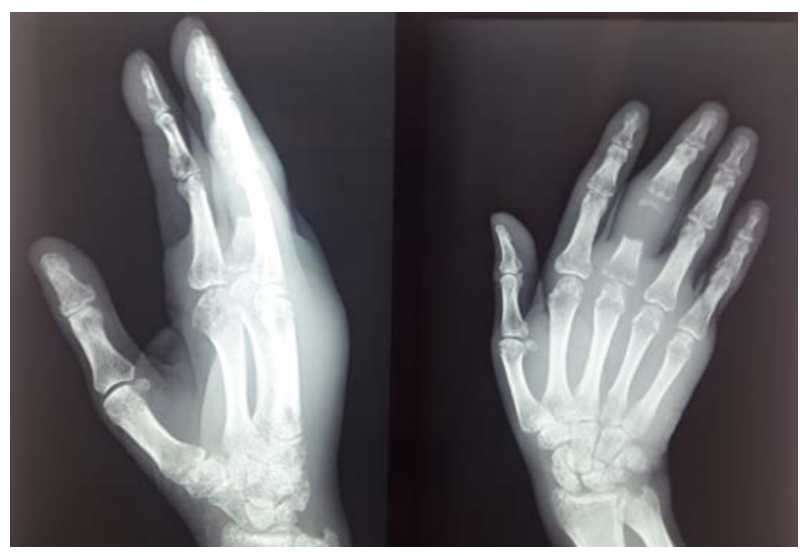

Figure 2. Hand x-ray of the $1^{\text {st }}$ patient at the $2^{\text {nd }}$ consultation.

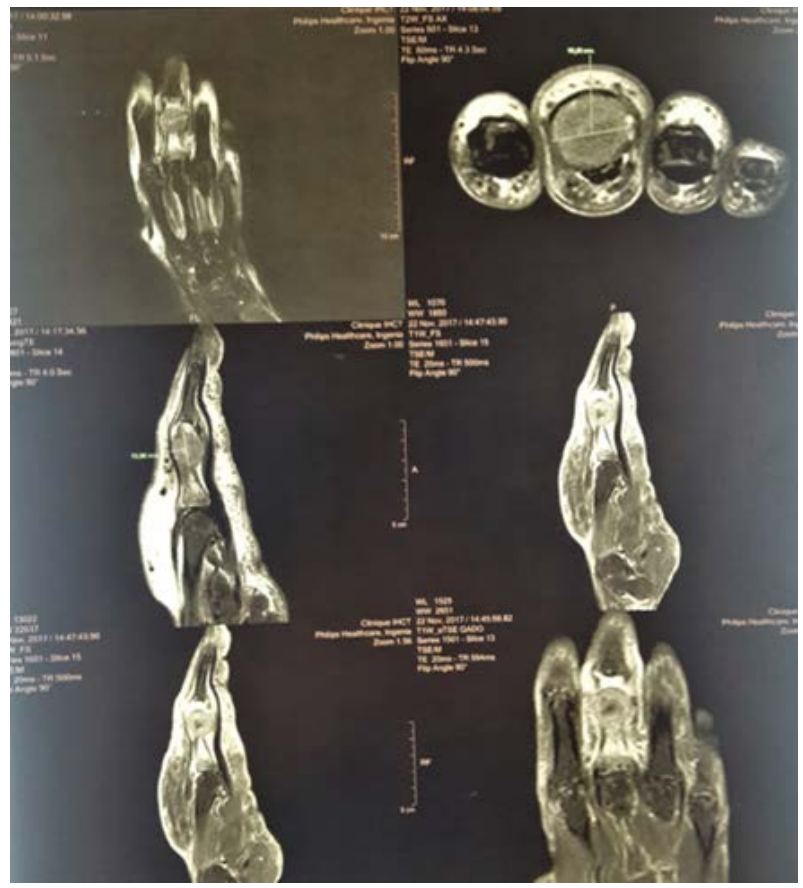

Figure 3. MRI of the $1^{\text {st }}$ patient. 


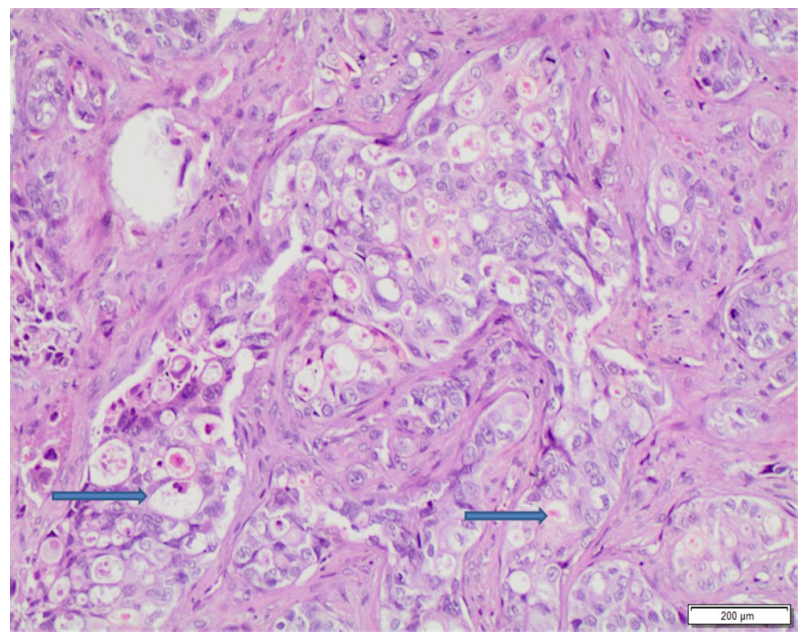

Figure 4. Metastasis of moderately differentiated adenocarcinoma. The proliferation is made up of massive and broad cellular spans hollowed out of glandular slits (arrow). (HE $\times 100$ coloring) $\left(1^{\text {st }}\right.$ patient).

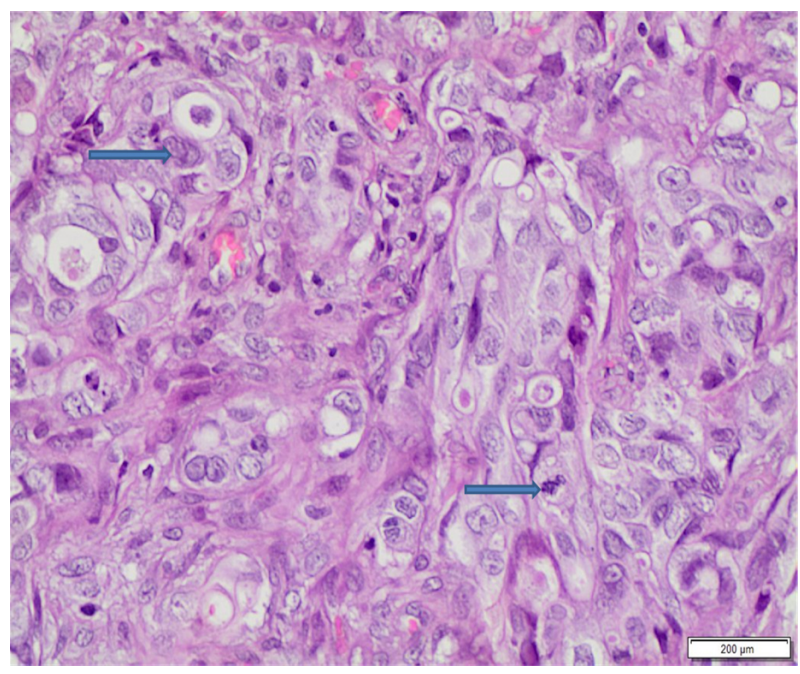

Figure 5. Malignant tumor proliferation of a moderately differentiated adenocarcinoma rich in atypia and mitosis (arrow). (HE $\times 200$ coloring) $\left(1^{\text {st }}\right.$ patient).
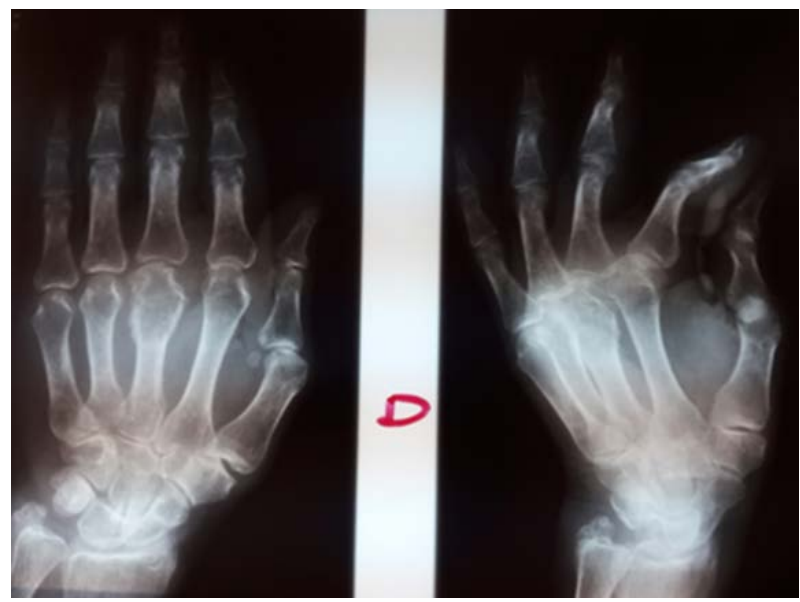

Figure 6. Hand $\mathrm{x}$-ray of the $2^{\text {nd }}$ patient (the $1^{\text {st }}$ one). 


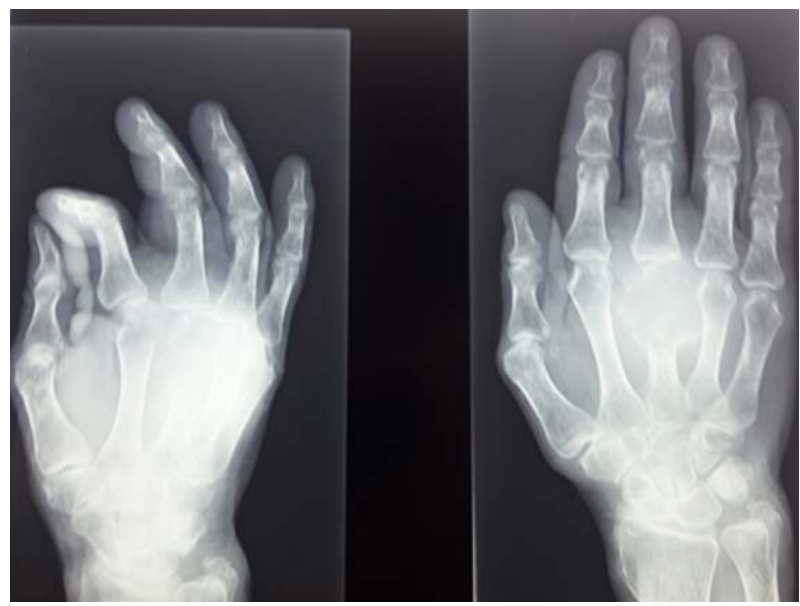

Figure 7. Hand $x$-ray of the $2^{\text {nd }}$ patient (the $2^{\text {nd }}$ one).

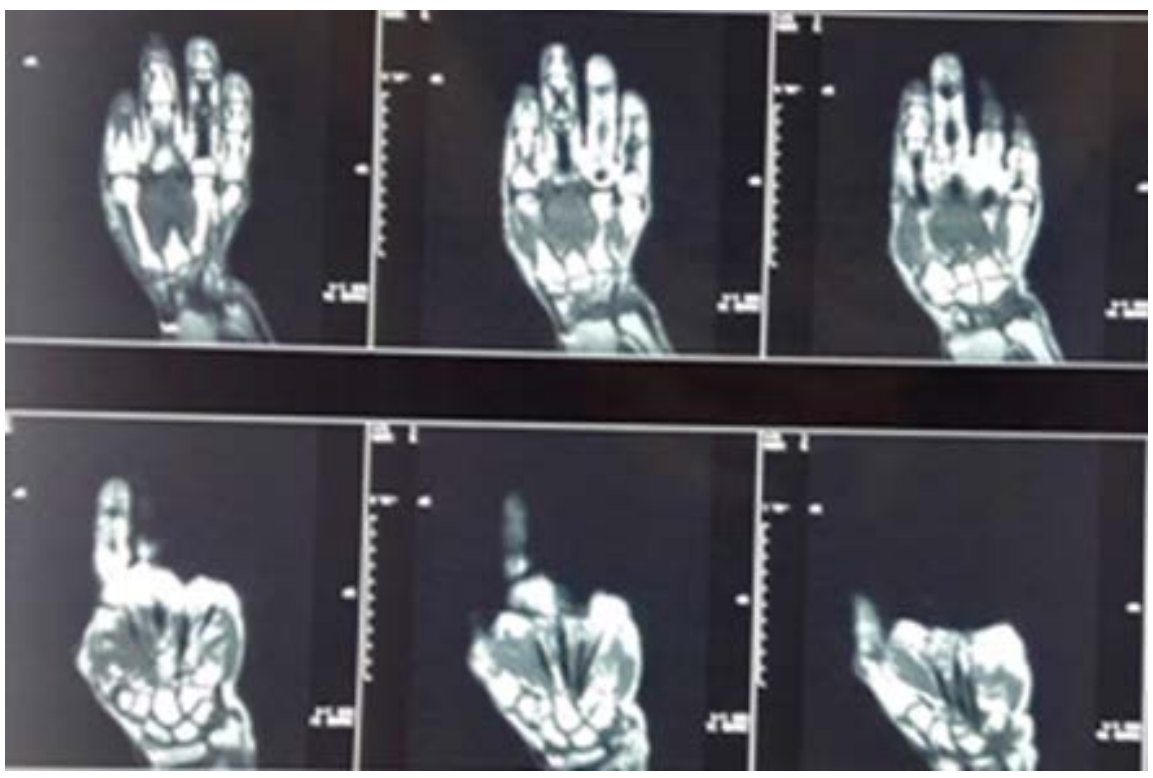

Figure 8 . MRI of the $2^{\text {nd }}$ patient.

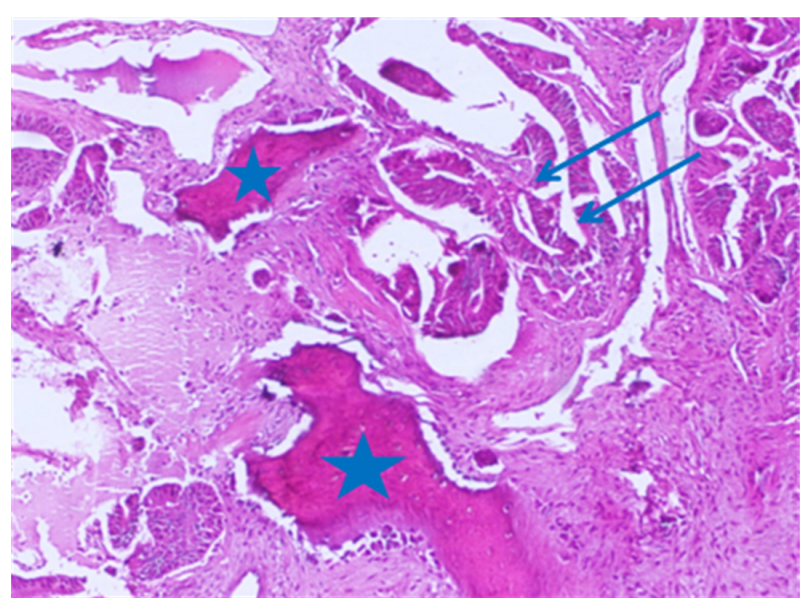

Figure 9. Bone tissue (star) invaded by metastatic glandular structures (arrows) (Low magnification, standard staining) ( $2^{\text {nd }}$ patient $)$. 


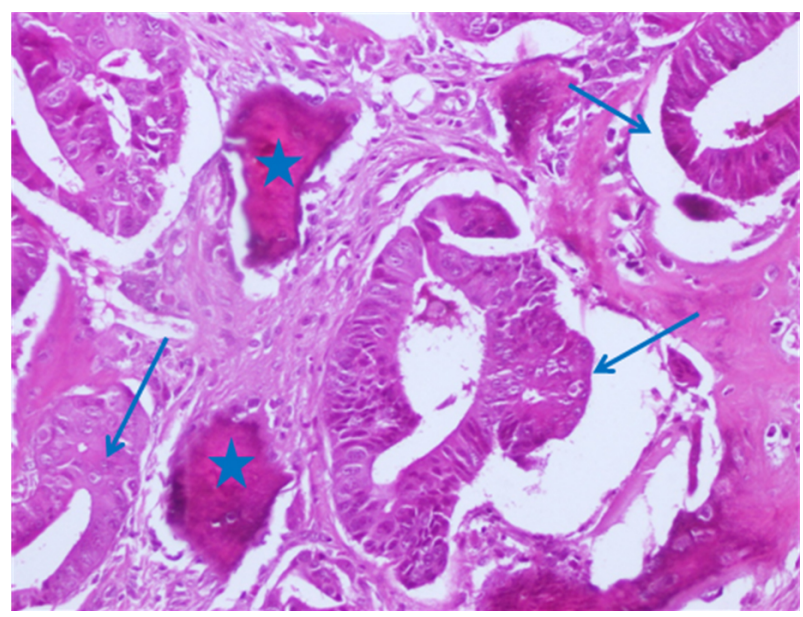

Figure 10. Metastatic glandular structures (arrows) encompassing bone sequesters (star) (High magnification, standard staining) ( $2^{\text {nd }}$ patient).

75,773 cancer patients, respectively. More recently, Munoz-mahamud [1] has collected only 57 cases of bone metastases reaching the hand from a review of the literature extended over 23 years (from 1993 to 2015) [1].

These acrometastases reveal the presence of cancer in $25 \%$ of cases [1]. This entity remains a subject of controversy because according to Bigot [5], bone metastases are indicative of cancer in sometimes more than half of the cases (15\% to $60 \%$ of the cases). This was the case with our first patient whose bone metastases revealed the existence of pulmonary adenocarcinoma. But it is more reasonable to believe that it is unusual to diagnose cancer from an acrometastasis because, on the one hand, of its rarity, and, on the other hand, because only $10 \%$ of acrometastases are symptomatic [1].

From a pathophysiological point of view, malignant cells preferentially reach bones with large capillary networks and significant blood flow. This explains the rarity of the peripheral bones and especially those of the hand, which are characterized by slowness of the blood flow circulating in a poorly developed network [6].

Clinically, our two patients present with a painful swelling in the hand without any other associated sign. But several clinical signs may be present in the initial examination of acrometastases. They usually present as pain, swelling, color change, ulceration, enlarged fingers, palpable masses, mechanical dysfunction or loss of function and pathological fractures [6]. This symptomatology is more frequently localized in the distal phalanges. The localization in metacarpal and the proximal phalanges is rarer. And it is extremely rare in carpal bones [7].

Furthermore, the finger most frequently affected by metastases is the thumb [6] [8]. This can be explained by the fact that the thumb and distal phalanx are more exposed to microtrauma thus generating prostaglandins and chemotactic factors contributing to the migration and fixation of malignant cells [6]. Moreover, Morris [8] recommends considering that any mechanical pain of the hand, particularly in the thumb, is a malignant process until proven otherwise. 
This clinical presentation may confuse other diagnoses such as hand infection, septic arthritis or osteomyelitis, rheumatic monoarthritis, algoneurodystrophy, tenosynovitis and gout disease [5] [6] [7].

In order to guide the diagnosis, a radiological assessment is necessary. On standard radiography, osteolysis is the usual radiological image. Additional MRI exploration is the rule to better explore bone involvement and adjacent areas. Finally, only the surgical biopsy and the pathology examination confirm the diagnosis.

The origin of this metastasis is primarily lung cancer (42\%), followed by the kidney (11\% to $14 \%$ ), the breast (11\%) and the digestive system (colon, stomach) [1] [2] [7] [9]. In contrast, rectal cancer is a very rare primitive, reported only once among 57 cases of bone metastases reported by Munoz-Mahamud. This localization concerned our second patient.

Acrometastases are characterized by a poor prognosis since they generally occur at a stage where the primitive has already been widely extended.

Treatment at this stage of the disease is generally palliative because of a short survival median varying from 3 to 6 months [10]. Surgery for these patients is very often difficult because of the risk of anesthesia on fragile ground. That was the case of our second patient. In our patients, the median survival was 4.5 months.

The therapeutic indications depend on the location of the metastasis, the number of lesions, the general condition of the patient and the primary condition. Pain treatment is the main component of therapeutic management. We can offer curettage followed by adjuvant radiotherapy, radiotherapy with or without chemotherapy [10] or chemotherapy alone in the proximal locations. Radical treatment based on marginal resection or amputation in distal locations can be offered [5], that was the case with our first patient.

\section{Conclusions}

A rapid and precise diagnosis of acrometastases is mandatory in order to avoid a delay in treatment. They are characterized by their rarity, by a poor prognosis and by an atypical clinic justifying the interest of a surgical biopsy especially since they are sometimes revealing of primary cancer.

The therapeutic component mainly concerns pain control in patients whose median survival is a few months. The management of such cases should be multifactorial with the cooperation of the surgeon with the radiologist, the pathologist and the oncologist to avoid unnecessary surgery.

\section{Conflicts of Interest}

The authors declare no conflicts of interest regarding the publication of this paper.

\section{References}

[1] Muñoz-Mahamud, E., Combalia, A., Carreño, A. and Arandes, J.M. (2017) Five 
Cases of Acrometastasis to the Hand from a Carcinoma and Review of the Literature. Hand Surgery \& Rehabilitation, 36, 12-16.

https://doi.org/10.1016/j.hansur.2016.10.211

[2] Keramidas, E. and Brotherston, M. (2005) Extensive Metastasis to the Hand from Undiagnosed Adenocarcinoma of the Lung. Scandinavian Journal of Plastic and Reconstructive Surgery and Hand Surgery, 39, 113-115.

https://doi.org/10.1080/02844310510006394

[3] Wu, K.K. and Guise, E.R. (1978) Metastatic Tumors of the Hand: A Report of Six Cases. The Journal of Hand Surgery, 3, 271-276. https://doi.org/10.1016/S0363-5023(78)80091-4

[4] Amadio, P.C. and Lombardi, R.M. (1987) Metastatic Tumours of the Hand. The Journal of Hand Surgery, 12, 311-316. https://doi.org/10.1016/S0363-5023(87)80299-X

[5] Bigot, P., Desbois, E., Benoist, N., Besnier, L. and Moui, Y. (2007) Isolated Pain Of the Hand Revealing a Metastatic Tumor of the Hand. Report of a Case. Chirurgie de la Main, 26, 300-302. https://doi.org/10.1016/j.main.2007.09.001

[6] Afshar, A., Farhadnia, P. and Khalkhali, H. (2014) Metastases to the Hand and Wrist: An Analysis of 221 Cases. The Journal of Hand Surgery, 39, 923-932. https://doi.org/10.1016/j.jhsa.2014.01.016

[7] Tzaveas, A., Paraskevas, G., Pazis, I., Dimitriadis, A., Kitsoulis, P., et al. (2008) Metastasis of Bronchogenic Carcinoma to the 5th Metacarpal Bone: A Case Report. Cases Journal, 1, Article No. 284. https://doi.org/10.1186/1757-1626-1-284

[8] Morris, G., Evans, S., Stevenson, J., Kotecha, A., Parry, M., et al. (2017) Bone Metastases of the Hand. Annals of the Royal College of Surgeons of England, 99, 563-567. https://doi.org/10.1308/rcsann.2017.0096

[9] Gilardi, R., Rosa, N.D., Pancaldi, G. and Landi, A. (2013) Acrometastasis Showing an Occult Lung Cancer. Journal of Plastic Surgery and Hand Surgery, 47, 550-552. https://doi.org/10.3109/2000656X.2012.748319

[10] Verma, Y., Chauhan, A.K. and Singh, H. (2013) Metastasis to Small Bone (Acrometastasis) of Hand Originating from Malignancy of Urinary Bladder: A Case Report. Journal of Clinical Case Reports, 3, 281. https://doi.org/10.4172/2165-7920.1000281 